\title{
Inversão de Temperaturas de Fundo de Poço para Determinação de Gradiente Geotérmico: Aplicação a Dados do Campo de Pineview (Utah, EUA).
}

\author{
Artur Benevides* (CPGG-UFBA), Amin Bassrei (CPGG-UFBA)
}

Copyright 2016, SBGf - Sociedade Brasileira de Geofísica

Este texto foi preparado para a apresentação no VII Simpósio Brasileiro de Geofísica, Ouro Preto, 25 a 27 de outubro de 2016. Seu conteúdo foi revisado pelo Comitê Ouro Preto, 25 a 27 de outubro de 2016. Seu conteúdo foi revisado pelo Comitê
Técnico do VII SimBGt, mas não necessariamente representa a opinião da SBGf ou de seus associados. É proibida a reprodução total ou parcial deste material para propósitos comerciais sem prévia autorização da SBGf.

\section{Abstract}

This work presents a geothermal study of Pineview oil field located in the state of Utah, USA. This study is characterized by two different stages: (1) the treatment of Bottom-Hole Temperature (BHT) data using the Horner's method in order to reduce the effects of disturbances related to the circulation of drilling mud and (2) the inversion of the BHT data to determine the geothermal gradient of some formations from a crosssection of Pineview field using Singular Value Decomposition (SVD). Initially, the inversion was tested in synthetic models to show its effectiveness. The inversion results revealed to be valid in the presence of enough information, in other words, when the linear system of equations is overdetermined or determined. The second step consisted in the inversion of real data and compared to values found by Deming and Chapman (1988) for the same region, resulted a reasonable accuracy in the determination of the geothermal gradients of some layers. Thus, the SVD method for geothermal data inversion demonstrated to work as an useful tool to estimate the geothermal gradients of layers.

\section{Introdução}

Diversos estudos geofísicos são realizados em uma bacia sedimentar, a maioria busca determinar regiões com potencial para produzir Petróleo. Dentre os estudos realizados pode-se destacar os Geotérmicos através de análises da temperatura, gradiente e fluxo geotérmico. Esses estudos podem trazer valiosas informações sobre a evolução tectônica da bacia, zonas de maturação do óleo, entre outras. Neste trabalho, busca-se através de medidas de TFP, de medidas de temperatura na superfície e informações sobre as espessuras das formações presentes em subsuperfície, determinar o gradiente geotérmico para cada formação perfilada.

As medidas de temperaturas no fundo do poço fazem parte da prática de perfilagem geofísica de poços e ocorrem um em curto período após cessada a perfuração do poço. Durante a perfuração do poço ocorre uma perturbação do campo térmico nas formações adjacentes, ocasionada fundamentalmente por dois fatores: o calor liberado pela broca durante a perfuração e a troca de calor entre a superfície e o fundo do poço, devido à circulação da Lama de perfuração. Portanto, para obter boa qualidade nos dados é necessário fazer uma correção nas medidas de BHT. O método utilizado para correção foi o Horner plot, conforme demonstra a seção correção de BHT. Os dados de temperatura na superfície foram obtidos através de medidas de estações meteorológicas da cidade de Salt Lake City, que fica próxima ao Campo de Pineview. Outro dado necessário ao modelo são as espessuras, para cada formação pelo qual passa um poço é necessário estabelecer uma espessura que somadas resulta na distância total entre o fundo do poço e a superfície.

\section{Área de Estudo}

O Campo de Pineview Figura 1 está localizado no noroeste do estado de Utah, nos Estados Unidos, a região está próxima ao limite deste estado com o estado de Wyoming. O campo faz parte do complexo de Absaroka, chamado de Absaroka thrust belt. O campo foi descoberto em 1975, e é um grande produtor de óleo.

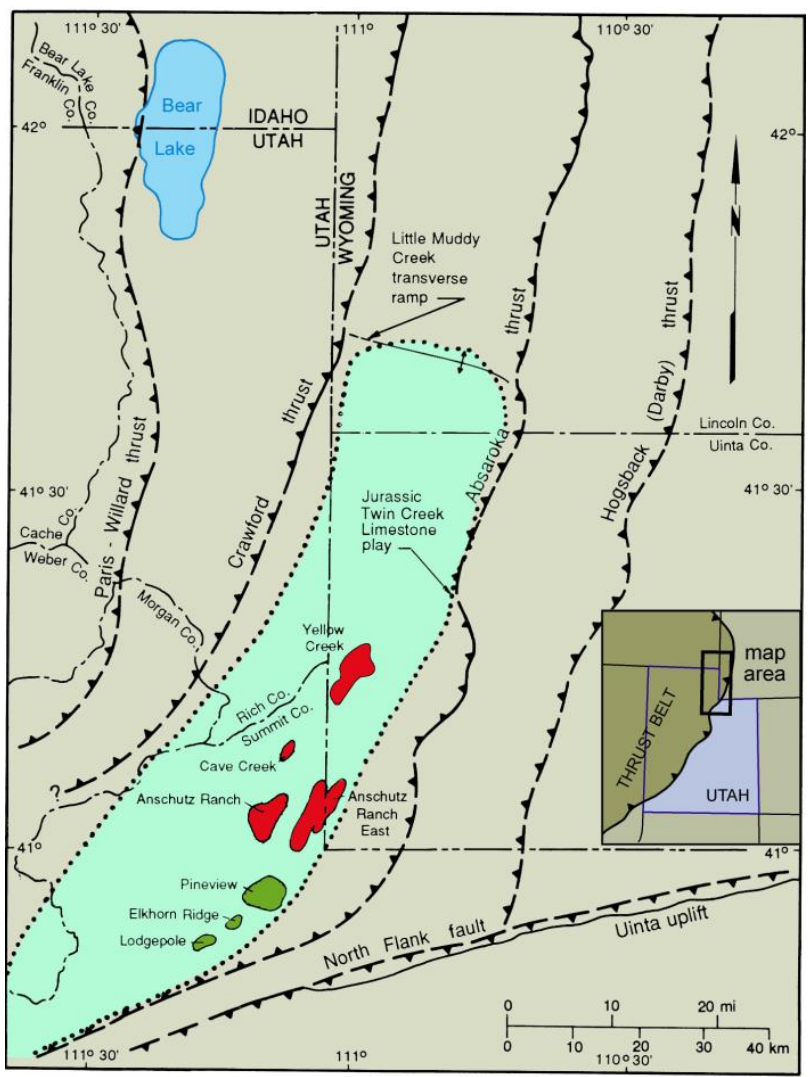

Figura 1. Mapa com a localização do Campo de Pineview, Utah, EUA. (Chidsey and Sprinkel, 2005) 


\section{Correção de BHT}

A correção das medidas de temperatura do fundo poço dá-se através do método proposto por Lachenbruch and Brewer (1957), que estima a temperatura verdadeira da formação através de uma extrapolação linear realizada utilizando-se a equação (1) quando temos no mínimo duas medidas de temperatura para uma mesma profundidade. O método de Horner é derivado do modelo de uma fonte linear de calor proposto por Bullard (1949). Este método foi utilizado também por Luheshi (1983), Deming e Chapman (1988), Cavalcante (2004), e é descrito pela equação abaixo:

$$
T\left(t_{s}\right)=T_{\infty}+\frac{Q}{4 \pi K}\left[E_{i}\left(\frac{-r^{2}}{4 \alpha\left(t_{s}+t_{c}\right)}\right)-E_{i}\left(\frac{-r^{2}}{4 \alpha t_{s}}\right)\right]
$$

\section{Teoria da Inversão}

O objetivo da inversão é a determinação de alguns parâmetros a partir de dados observados, ou, em face das limitações inevitáveis nos processos de medições reais, o máximo de informações possíveis acerca de tais parâmetros (Bassrei,1990).

A técnica da modelagem direta calcula os dados decorrentes de um determinado fenômeno modelado, conhecendo-se os parâmetros do modelo, ou seja, partese dos parâmetros para a obtenção dos dados.

O problema inverso é relativamente mais complicado, uma vez que, em situações reais podemos obter infinitos modelos que se adequam aos mesmos dados. $O$ objetivo do problema inverso é determinar qualquer entrada ou o sistema que causa as medidas da saída.

Uma vez obtido os parâmetros do modelo (estimado), utilizamos os parâmetros de dados para analisar as propriedades deste modelo e o que ele preserva do modelo real, tal como erro e ruídos.

\section{Problema Inverso}

Para inversão de temperatura de fundo de poço

formulamos o problema seguinte:

$$
\boldsymbol{T}_{\Delta}=Z \boldsymbol{g},
$$

sendo

e

$$
\begin{gathered}
\boldsymbol{T}_{\Delta}=\left[T_{\Delta 1}, T_{\Delta 2}, T_{\Delta 3}, \ldots, T_{\Delta M}\right]^{T}, \\
\boldsymbol{g}=\left[g_{1}, g_{2}, g_{3}, \ldots, g_{N}\right]^{T},
\end{gathered}
$$

onde $\boldsymbol{T}_{\Delta}$ representa um vetor de dados medidos

$$
T_{\Delta M}=T_{b h t M}-T_{0},
$$

g representa um vetor contendo os gradientes geotérmicos a ser determinado pela inversão, $Z(M, N)$ é uma matriz que relaciona os vetores $g$ e $\boldsymbol{T}_{\Delta}$. A matriz $Z$ contêm as espessuras das formações.

Supondo a matriz $Z$ conhecida, pode-se resolver o sistema da equação (2), utilizando uma matriz inversa, ou seja,

$$
\boldsymbol{g}=Z^{-1} \boldsymbol{T}_{\Delta}
$$

Deste modo, pode-se estimar os valores dos gradientes de cada camada utilizando uma inversão de dados.

O método que será utilizado para a solução do problema inverso é o da decomposição por valores singulares $S V D$.

\section{Decomposição SVD}

A Decomposição em valores singulares ou SVD, é uma técnica empregada para a simplificação de matriz associada a uma transformação linear. As matrizes que representam problemas geofísicos são na sua maioria retangulares, ou seja, possuem mais icógnitas a serem determinadas em relação a quantidade de equações ou informações. Nessas condições pode-se determinar a pseudo-inversa de uma determinada matriz $\boldsymbol{Z}$ utilizandose o SVD.

Seja $Z$ uma matriz $M x N$. A sua matriz $Z^{+}$será a sua pseudo-inversa, ou inversa generalizada de MoorePenrose, agora de dimensões $(N x M)$. Para calcular a inversa utiliza-se a decomposição de Lanczos (1961).

Supondo uma matriz retangular $G_{M x N}$ de posto $k$, sua $S V D$ é da seguinte forma:

$$
Z=U \Sigma V^{T}
$$

tal que: $U_{M \times M}$ é a matriz que contém os autovetores ortonormalizados de $Z Z^{T}, \Sigma_{M x N}$ é a matriz que contém as raízes quadradas dos autovalores de $Z^{T} Z$, denominados valores singulares, sendo estes colocados em ordem decrescente, ou seja, $\sigma_{1} \geq \sigma_{2} \geq \cdots \geq \sigma_{k}>0, V_{N X N}$ é a matriz que contém os autovetores ortonormalizados de $Z^{T} Z$.

Então a sua Pseudo-inversa é:

$$
Z^{+}=V \Sigma^{+} U^{T},
$$

onde $\Sigma^{+}(N x M)$, é a matriz que contém os inversos dos valores singulares não nulos de $Z$ da seguinte forma:

$$
\Sigma^{+}=\left[\begin{array}{cc}
E & 0 \\
0 & 0
\end{array}\right],
$$

onde $E$ é a matriz $k x k$ cujo o $i$-ésimo elemento diagonal é $e_{i i}=e_{i}^{-1}$ para $1 \leq i \leq k$ :

$$
E=\left[\begin{array}{cccc}
\sigma_{1}{ }^{-1} & 0 & \cdots & 0 \\
0 & \sigma_{2}{ }^{-1} & \cdots & 0 \\
\vdots & \vdots & \ddots & \vdots \\
0 & 0 & \cdots & \sigma_{k}{ }^{-1}
\end{array}\right] .
$$

\section{Modelagem direta}

Para averiguar a eficácia da inversão de dados por $S V D$, nesta seção é realizado o cálculo direto de $\boldsymbol{T}_{\Delta} \mathrm{com}$ a equação (2) utilizando valores de gradientes verdadeiros propostos para 2 modelos com 3 camadas. 


\section{Modelo Sintético (I)}

Utilizando dados de 3 poços: espessuras e gradiente das camadas perfiladas, podemos determinar $\boldsymbol{T}_{\Delta}$, as características do modelo pode ser observada na Figura 2 e os valores estão presentes na Tabela 1.

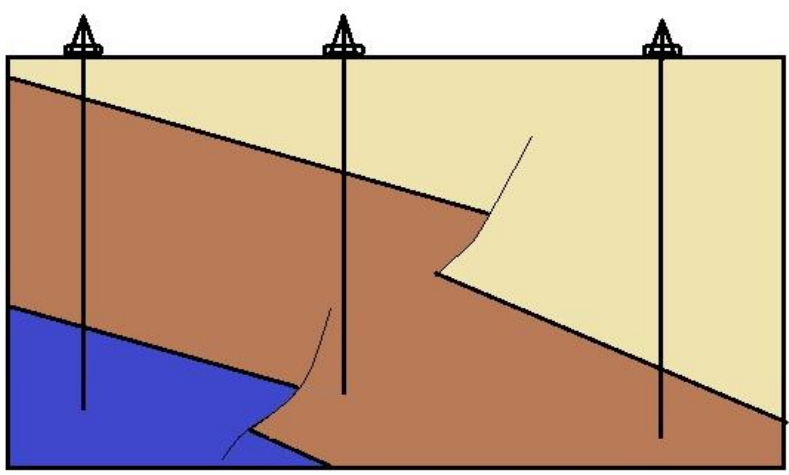

Figura 2: Seção geológica exemplificando o modelo sintético (I).

Tabela 1. Espessuras e gradientes das formações referente ao modelo sintético (I).

\begin{tabular}{|c|c|c|c|c|}
\hline formações & $P 1(\mathrm{~m})$ & $P 2(\mathrm{~m})$ & $P 3(\mathrm{~m})$ & $g^{\text {ver }}\left({ }^{\circ} \mathrm{C} / \mathrm{m}\right)$ \\
\hline$\square$ & 10.0 & 30.0 & 70.0 & 0.0196 \\
\hline$\square$ & 56.0 & 50.0 & 17.0 & 0.0235 \\
\hline$\square$ & 15.0 & 0.0 & 0.0 & 0.0257 \\
\hline
\end{tabular}

As diferenças de temperatura obtidas são:

$\boldsymbol{T}_{\Delta}=\left[\begin{array}{lll}1.89 & 1.76 & 1.77\end{array}\right]^{T}\left({ }^{\circ} \mathrm{C}\right)$

\section{Modelo Sintético (II)}

Este modelo possui mais informação, pois tem um poço a mais em relação ao modelo anterior. A Figura 3 exemplifica o modelo e seus dados estão presentes na Tabela 2. Busca-se determinar também $\boldsymbol{T}_{\Delta}$ utilizando equação (2).

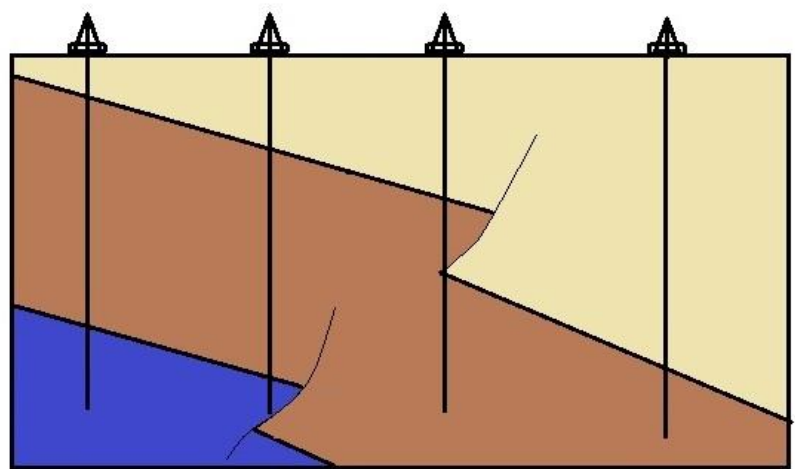

Figura 3. Seção geológica exemplificando o modelo sintético (II).
As diferenças de temperatura obtidas são:

$\boldsymbol{T}_{\Delta}=\left[\begin{array}{llll}1.89 & 1.92 & 1.95 & 1.77\end{array}\right]^{T}\left({ }^{\circ} \mathrm{C}\right)$

Tabela 2. Espessuras e gradiente das formações referente ao modelo (II).

\begin{tabular}{|c|c|c|c|c|c|}
\hline formações & $P 1(m)$ & $P 2(m)$ & $P 3(m)$ & $P 4(m)$ & $\begin{array}{c}g^{\text {ver }} \\
\left({ }^{\circ} \mathrm{C} / m\right)\end{array}$ \\
\hline$\square$ & 10.0 & 28.0 & 40.0 & 70.0 & 0.0196 \\
\hline$\square$ & 56.0 & 50.0 & 50.0 & 17.0 & 0.0235 \\
\hline$\square$ & 15.0 & 8.0 & 0.0 & 0.0 & 0.0257 \\
\hline
\end{tabular}

\section{Inversão de dados sintéticos}

A aplicação em sintéticos nos permite medir o tamanho do erro entre o $\boldsymbol{g}^{\text {ver }}$ e $\boldsymbol{g}^{\text {est }}$ pois, temos mais controle dos parâmetros do modelo. As Tabelas 1 e 2 apresentam os valores verdadeiros para os gradientes das camadas. Aplicando o método $S V D$ na matriz das espessuras $Z$, obtém-se a sua pseudo-inversa $Z^{+}$, e posteriormente utilizando a equação (4), calcula-se os valores de $g^{e s t}$.

No modelo (I), a matriz é $Z(3,3)$, o sistema de equações é um caso determinado, o cálculo da inversa é mais prático em relação a matrizes retangulares, a matriz $Z(3,3)$ tem posto completo e sua pseudo-inversa é idêntica à inversa clássica. Os valores obtidos na inversão são:

$$
\boldsymbol{g}^{\text {est }}=\left[\begin{array}{lll}
0.0195 & 0.0228 & 0.0251
\end{array}\right]\left({ }^{\circ} \mathrm{C} / \mathrm{m}\right) .
$$

O erro RMS (\%) entre os $\boldsymbol{g}^{\text {ver }}$ e $\boldsymbol{g}^{\text {est }}$ foi de 2.320.

No modelo (II) a matriz é $Z(4,3)$, o sistema linear de equações é classificado como um caso sobredeterminado de posto $k=3$, sua pseudo-inversa não é idêntica à inversa clássica como no modelo anterior. Os valores obtidos na inversão são:

$\boldsymbol{g}^{\text {est }}=\left[\begin{array}{lll}0.0201 & 0.0224 & 0.0255\end{array}\right]\left({ }^{\circ} \mathrm{C} / \mathrm{m}\right)$.

O erro RMS (\%) entre $\boldsymbol{g}^{\text {ver }}$ e $\boldsymbol{g}^{\text {est }}$ foi de 2.842.

Os gradientes obtidos com a inversão tanto para o caso determinado quanto para o sobredeterminado possuem boa precisão, podemos observar pelos valores do erro RMS que as medidas estimadas estão bem próximas das verdadeiras. Demonstrando assim a eficácia do método de inversão para resolução do problema inverso proposto.

\section{Inversão de Dado Real}

Depois de verificado a eficácia do método em modelos sintéticos, busca-se aplicá-lo na solução de problemas geotérmicos reais.

A Inversão será aplicada na determinação de gradientes geotérmicos de formações geológicas presentes no Campo de Óleo de Pineview. A Figura 7 mostra uma seção geológica do campo de Pineview. Nesta seção, 
pode-se visualizar a disposição dos poços e das formações, pode-se perceber pela disposição das formações que o ambiente geológico é complexo, com diversas estruturas geológicas ocasionadas por esforços tectônicos.

As falhas são de empurrão, devido a movimentos tectônicos compressivos. Essas Falhas representam deslocamento (Não envolve rocha do embasamento), que são estilos de deformações compressionais. Os empurrões em sua maioria tem tendência para as direções norte-nordeste. As bordas destas falhas são da forma Lístrica e estruturalmente complexa, com numerosas dobras e falhas secundárias (Chidsey e Sprinkel, 2005).

$\mathrm{Na}$ Figura 6 apresentamos um perfil estratigráfico do Campo de Pineview que descreve as características litológicas de cada formação.

Para determinação dos gradientes geotérmicos de cada formação, precisamos dos valores de $T_{B H T}, T_{0}$ e fazer a inversão da matriz das espessuras de cada camada perfilada.

As $T_{B H T}$ referente aos 8 poços estão presentes na Figura 7.

$T_{0}$ é uma temperatura média da superfície para região. Utilizando dados meteorológicos foi adotada uma temperatura média de $12{ }^{\circ} \mathrm{C}$ para superfície da região.

Com essas duas informações, temos o $\boldsymbol{T}_{\Delta}$ de cada poço. As espessuras que compõem a $Z_{i j}$ Podem ser observadas na Tabela 3.

Nesta inversão, contamos com um sistema sobredeterminado, ou seja, possuímos mais informações de poço em relação à quantidade de formações que precisamos determinar os gradientes.
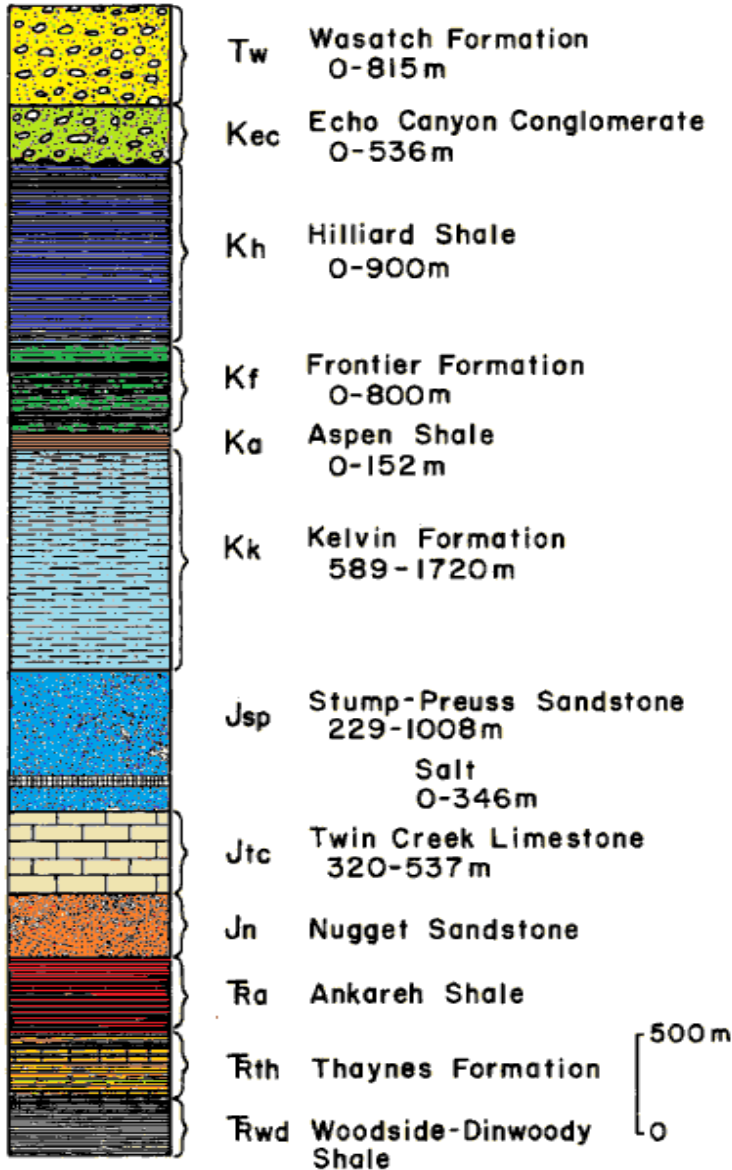

Figura 6. Coluna estratigráfica do campo de Pineview. (Modificado de Deming e Chapman, 1988)

Tabela 3: Espessuras das Formações para os 8 poços.

\begin{tabular}{|c|c|c|c|c|c|c|c|c|}
\hline formação & Poço 1 (m) & Poço 2 $(\mathrm{m})$ & Poço 3 $(\mathrm{m})$ & Poço 4 $(\mathrm{m})$ & Poço 5 $(\mathrm{m})$ & Poço 6 $(\mathrm{m})$ & Poço 7 (m) & Poço 8 (m) \\
\hline Tw & 0.0 & 350.0 & 350.0 & 500.0 & 525.0 & 525.0 & 575.0 & 650.0 \\
\hline Kec & 175.0 & 170.0 & 170.0 & 375.0 & 350.0 & 350.0 & 325.0 & 325.0 \\
\hline kk & 1700.0 & 550.0 & 1350.0 & 1125.0 & 1100.0 & 1100.0 & 1175.0 & 725.0 \\
\hline Jsp & 550.0 & 0.0 & 500.0 & 325.0 & 275.0 & 275.0 & 175.0 & 775.0 \\
\hline Jtc & 350.0 & 0.0 & 350.0 & 350.0 & 350.0 & 350.0 & 350.0 & 450.0 \\
\hline Jn & 175.0 & 0.0 & 250.0 & 250.0 & 0.0 & 300.0 & 300.0 & 125.0 \\
\hline
\end{tabular}




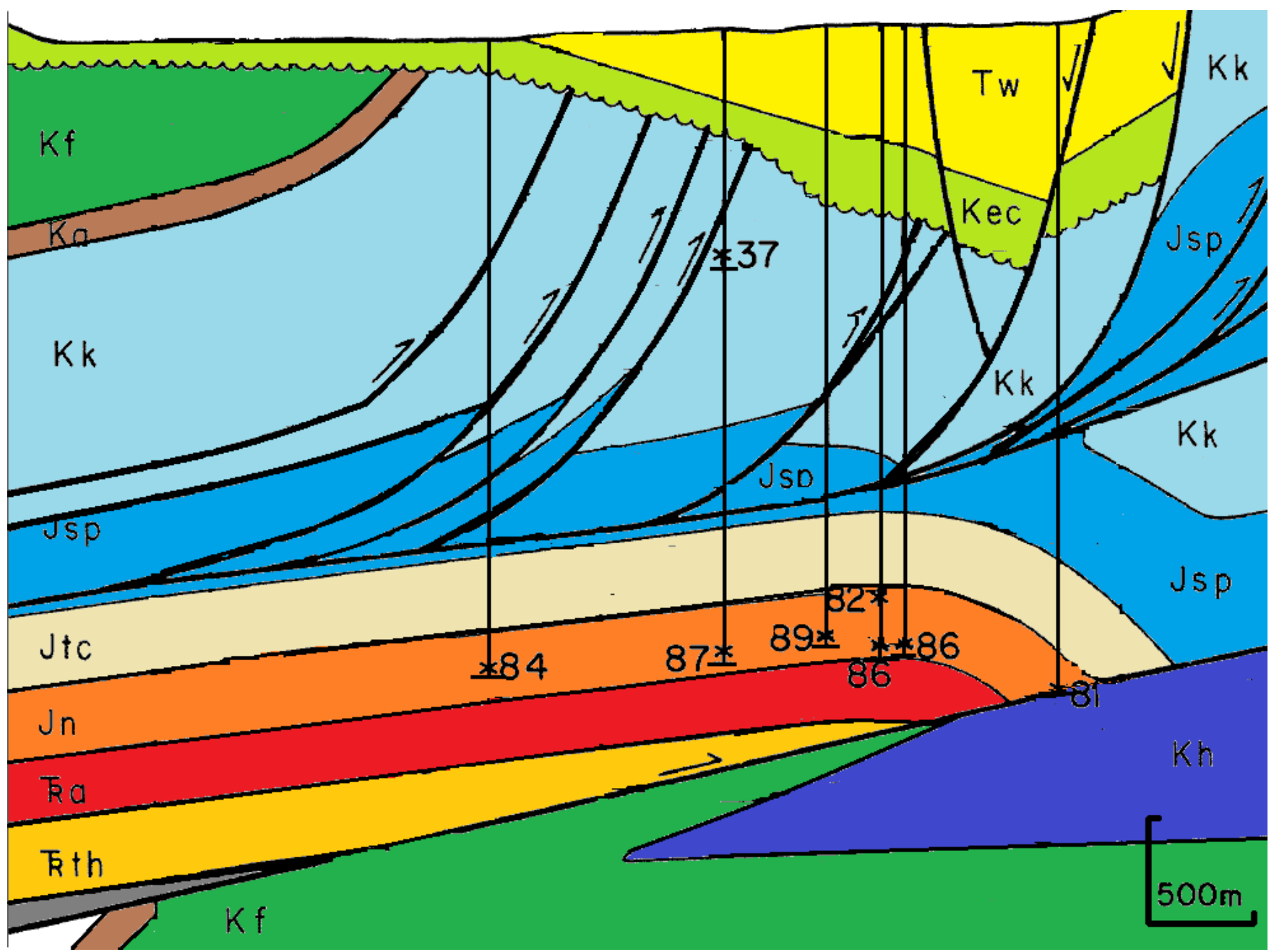

Figura 7: Seção transversal do Campo de Pineview. (Modificado de Deming e Chapman, 1988).

\section{Resultados}

Como resultado da inversão, obtivemos os valores dos gradientes geotérmicos para cada formação Tabela 4 .

Tabela 4. Resultado da Inversão. Gradientes geotérmicos das 6 formações perfiladas $\boldsymbol{g}^{\text {est }}$, e os gradientes obtidos por Deming e Chapman (1988) utilizando norma $\mathbf{l 1}$ e norma $\mathbf{l 2}$.

\begin{tabular}{|c|c|c|c|}
\hline formação & $\begin{array}{c}\boldsymbol{g}^{\mathbf{e s t}}\left({ }^{\circ} \mathrm{C}\right. \\
\mathbf{k m})\end{array}$ & $\boldsymbol{g} \mathbf{l 1}\left({ }^{\circ} \mathrm{C} / \mathbf{k m}\right)$ & $\begin{array}{l}\mathbf{g} \mathbf{l 2}\left({ }^{\circ} \mathrm{C}\right. \\
\mathbf{k m})\end{array}$ \\
\hline Tw & 21.8 & 18.6 & 22.2 \\
\hline $\mathbf{K e C}$ & 21.0 & 17.2 & 20.1 \\
\hline $\mathbf{K k}$ & 26.2 & 26.4 & 27.5 \\
\hline $\mathbf{J s p}$ & 13.1 & 13.5 & 14.8 \\
\hline Jtc & 34.5 & 42.5 & 39.1 \\
\hline Jn & 32.5 & 38.6 & 22.8 \\
\hline
\end{tabular}

\section{Discussão e Conclusões}

O resultado obtido na inversão apresentam valores coerentes do ponto de vista físico e semelhantes aos encontrados por Deming e Chapman (1988), na determinação dos gradientes para as formações Tw, Kec, Kk, Jtc e Jn como podemos observar na tabela 4. Deming e Chapman (1988) determinaram os gradientes utilizando o método da norma $l 2$ e norma $l 1$.

O gradiente determinado para formação Jsp apresentouse com um valor relativamente baixo, isso pode ser justificado pela presença de uma camada de sal no meio da formação Jsp, veja perfil estratigráfico na Figura 6. Segundo Silva (2010), o Sal está entre as rochas mais condutivas das bacias sedimentares, com $K=$ $6,1 \mathrm{Wm}^{-1}{ }^{\circ} \mathrm{C}^{-1}$ a $20^{\circ} \mathrm{C}$, isto é explicado pela baixa porosidade e consequente ausência de fluido de saturação. 


\section{Agradecimentos}

O Autor agradece ao Conselho Nacional de Desenvolvimento Científico e Tecnológico (CNPq) pelo apoio financeiro, ao Centro de Pesquisa em Geofísica e Geologia (CPGG/IGEO/UFBA) pela infraestrutura e a Financiadora de Estudos e Projetos (FINEP) pela oportunidade da pesquisa.

\section{Referências}

Bassrei, A., 1990, Inversão de dados Geofísicos Unidimensionais através da Entropia Relativa Mínima. Tese de Doutorado. Universidade Federal da Bahia. Salvador.

Bullard, E. C., 1947, The time necessary for a bore hole to attain temperature equilibrium: Monthly Notices Royal Astronomical Society, Geophysical Supplement, v. 5, no. 5 , p.127-130

Chidsey, T. C. Jr., Sprinkel, D. A., Major Oils Plays in Utah and Vicinity. Utah Geological Survey. Salt Lake City.

Deming, D., Chapman, S. D., 1988. Inversion of Bottomhole Temperature data: The Pineview field, UtahWyoming thrust belt. Geophysics, 53: 707-720.

Cavalcante, A. G., Argollo, R. M., Carvalho, H. S., 2004, Correções de dados de temperatura de fundo de poço (TFP), Revista Brasileira de Geofísica.

Eldorado County Weather http://www.eldoradocountyweather.com/current/climate/n ationalclimate.html, acessado em: 24de Abril de 2016. às $20 \mathrm{~h}$.

Lachenbruch, A. H., Brewer, M. C., 1959, Dissipation of the temperature effect of drilling a well in Arctic Alaska. U. S. Geol. Survey Bull., 1083-C: 73-109.

Luheshi, M. N., 1983, Estimation of formation temperature from borehole measurements: Geophysical Journal [Royal Astronomical Society], v. 74, p. 747-776.

Menke, W. 1984. Geophysical Data Analysis: Discrete Inversion Theory. Academic Press Inc.

Silva, F. C., 2010. Condutividade Térmica, Difusividade Térmica e Calor específico em rochas ígneas e metamórficas: Comportamento Isotrópico e Anisiotrópico. Trabalho de Graduação, Universidade Federal da Bahia. Salvador.

Clima da região de Salt Lake City: http://www.climatezone.com/climate/united-states/utah/salt-lake-city/.

Acessado em: 24 de Abril de 2016, ás 20h. 